\title{
Central serous chorioretinopathy: a seasonal variation?
}

\author{
GARY H. CASSEL, GARY C. BROWN, AND WILLIAM H. ANNESLEY \\ From the Retina Service, Wills Eye Hospital, 9th and Walnut Streets, Philadelphia, PA 19107, USA
}

SUMmARY A review of 345 consecutive cases of patients under the age of 40 years with central serous chorioretinopathy seen between 1969 and 1979 was performed in order to define temporal patterns of occurrence. The monthly distribution of cases significantly differed $(p<0.01)$ from an expected random distribution. Although a statistical trend analysis failed to confirm a definite seasonal variation $(p>0 \cdot 01)$, an increased number of cases were seen in March and April.

First recognised by von Graefe in 1866 , central serous chorioretinopathy (CSCR) was initially referred to as central recurrent retinitis.' Since that time many other terms have been applied to this disease, including central angiospastic retinopathy and idiopathic flat detachment of the macula.' The clinical presentation is characterised by a transient episode of serous detachment of the retina in the macular region. Fluorescein angiography often reveals a disturbance at the level of the retinal pigment epithelium and leakage of dye from the choroid into the subretinal space.'

Observations in the past have provided several interesting epidemiological facts. CSCR ?in!y affects people between the ages of 20 and 41 ' years.' It has been recognised to occur more often in males than in females in a ratio ranging from $3: 1$ to $6: 1 .^{23}$ Although usually unilateral, it may be bilateral. Resolution is most often within three to four months,' and the recurrence rate is estimated to be $40 \%$ to $50 \%$ within eight years. ${ }^{3+}$ The disease is more prevalent in Japan than in the Western Hemisphere. Stress and unusual emotional upset have been implicated as important factors in its occurrence,' though no specific systemic diseases have been associated with it.

Despite these characteristics and the speculations of many authors on the aetiology of CSCR, the disease remains an enigma. A clinical impression led us to suspect a seasonal variation in the occurrence of cases. The purpose of this analysis was to study the occurrence of CSCR for a seasonal trend and hopefully provide additional clues to its aetiology.

Correspondence to Dr Gary Brown, 910 E Willow Grove Avenuc. Philadelphia. PA 19118. USA.

\section{Materials and methods}

The cases of all patients with central serous chorioretinopathy seen on the Retina Service of Wills Eye Hospital between January 1969 and December 1979 were included in the analysis. CSCR was defined as an isolated serous elevation of macular retina unassociated with subretinal blood, optic nerve head pathology, or evidence of other retinochoroidal or systemic disease. Pinpoint leakage of dye at the level of the retinal pigment epithelium, with subsequent pooling in the subsensory retinal space during fluorescein angiography, was also used to help make the diagnosis.

Our patients were referred by other physicians, as is characteristic of most large teaching hospitals providing care to an urban/metropolitan area. This can be advantageous when studying a disease of infrequent occurrence such as this. The referral of cases allows for an accumulation of information and for the subsequent analysis of the data. A delay in referral, however, may interfere with the estimation of the time of onset of an illness. However, with a few exceptions all patients in our series were seen within one month of the onset of visual symptoms.

Central serous chorioretinopathy usually occurs in middle-aged people, although older people have also been found to have it. The disease may be confused with serous detachment of the retina secondary to an unsuspected subretinal neovascular membrane, which usually occurs in older people. We therefore decided to limit our analysis to patients 40 years of age and younger to lessen the likelihood of this confusion in diagnosis. 
The total number of patients aged 40 years and under observed with an episode of CSCR during the study period was 345 . Only patients who were seen on the Retina Service for the first time were included in the analysis. The $\chi^{2}$ test and seasonal trend analysis as described by Edwards were used in the investigation.

Edwards's method has been proposed for the estimation of cyclic trends. 5 " A discussion of its advantages over other analyses of trend can be found elsewhere. The tabulations proposed by Edwards are especially appropriate for large samples $(n>100)$ and therefore were employed.

\section{Results}

The male to female ratio in our population (6:1) was very similar to that reported by other authors. ${ }^{3}$ We also derived confidence in our data when we noted that the annual numbers of cases seen in the years 1969 to 1979 (Table 1) were relatively consistent, with variations probably due to chance alone $(p>0 \cdot 1)$.

The result of the $\chi^{2}$ test for significance on the monthly distribution of cases as observed between 1969 to 1979 (Table 2) was 25.53, with 11 degrees of freedom. This is significant at the $\mathrm{p}<0.01$ level, suggesting that the distribution of cases is different from what would be expected by chance alone. As analyses taking into account the exact number of days for each month have been thought to be unnecessary and to have little effect on the results,

Table 1 Numbers of cases per year of central serous chorioretinopathy seen at the Wills Eye Hospital Retina Service (1969-79)

\begin{tabular}{llll}
\hline Year & Cases & Year & Cases \\
\hline 1969 & 30 & 1975 & 39 \\
1970 & 33 & 1976 & 34 \\
1971 & 31 & 1977 & 25 \\
1972 & 41 & 1978 & 15 \\
1973 & 35 & 1979 & 29 \\
1974 & 33 & Total & 345 \\
\hline
\end{tabular}

Table 2 Monthly distribution of cases $(n)$ of central serous chorioretinopathy (1969-79)

\begin{tabular}{llll}
\hline Month & $n$ & Month & $n$ \\
\hline January & 25 & August & 33 \\
February & 26 & September & 27 \\
March & 39 & October & 23 \\
April & 44 & November & 35 \\
May & 30 & December & 23 \\
June & 27 & & \\
July & 13 & Total & 345 \\
\hline
\end{tabular}

the statistic was calculated using an average of 30 days per month.

The distribution of cases appeared to suggest a peak between March and April (Fig. 1). Therefore a trend analysis was conducted on the 12-month totals. A $\chi^{2}$ value of 3.7 with 2 degrees of freedom was obtained, which was not significant at the $p>0 \cdot 1$ level.

A statistically significant seasonal trend could not be demonstrated in our cases despite the appearance of an increased occurrence in March and April (Fig. 1). The distribution also does not appear to be a function of the overall referral pattern of patients to the Retina Service at the Wills Eye Hospital. A separate trend analysis using Retina Service information representative of the monthly distribution of all people seen on this service between 1969 and 1979 showed the peak volume to occur between June and July $(p<0.05)$. Therefore the distribution shown by our series of CSCR patients is significantly different from that expected on chance alone and is not explicable by a seasonal variation or by association with patient flow patterns to the Retina Service.

\section{Discussion}

The aetiology of CSCR is not known. 'Stress' and various emotional factors have been suggested as playing a role in its development.' Investigators have also speculated on an allergic mechanism.' The seasonal variation of certain environmental allergens has been proposed to influence its occurrence. On this hypothesis CSCR might be expected to have a statistically significant seasonal variation. A marked decrease in its prevalence would also be expected during the months when seasonally related allergens are not commonly present in the environment. Our findings, however, do not support this hypothesis. The distribution of our cases reveals a slight increase in the months of March and April and

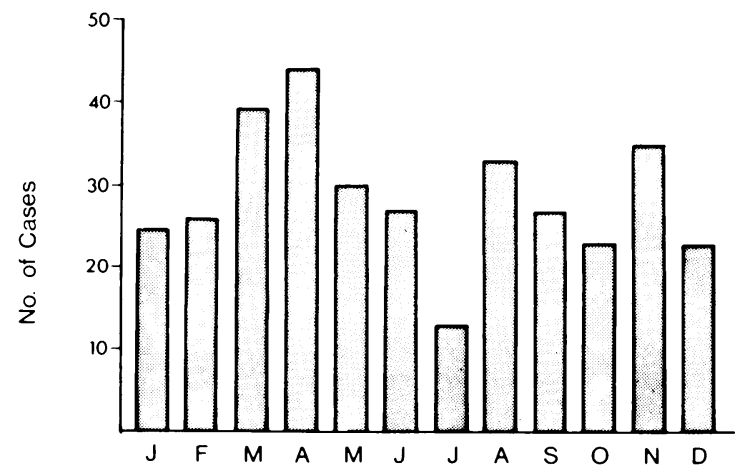

Fig. 1 Monthly distribution of cases of central serous chorioretinopathy, 1969-79. 
again in the colder months of the year, but we could not demonstrate statistically significant peaks of seasonal occurrence.

The possibility of an infectious aetiology has also been proposed. ${ }^{\prime}$ Sie-Boen-Lian searched for a viral mechanism in CSCR. ${ }^{7}$ He succeeded in cultivating typical viral elementary bodies in ova inoculated with the blood of patients with CSCR, and subsequently proposed that the disease may be the result of a subclinical viraemia in affected individuals. Certain endemic viral infections are known to have a seasonal pattern. ${ }^{*}$ These infections can persist in a community, causing sporadic cases during the year, with slight peaks due to seasonal fluctuations. Rhinovirus and corona virus are endemic infections which commonly produce cold-like illnesses in adults. Both these agents occur throughout the year, with peaks in the spring and autumn." In temperate regions certain types of adenoviruses $(1,2$, and 5$)$ are also endemic, but somewhat more frequent in spring, early summer, and late autumn." Although the possibility of a viral aetiology for CSCR is interesting, our data can neither prove nor disprove it.

Histopathological studies of the acute phase of the illness are generally not readily available, and therefore we can only speculate on the pathogenesis of this entity. Our review has confirmed the impression of a non-random monthly distribution of cases referred to our clinic. This distribution, however, does not demonstrate a significant seasonal variation. At this time the reasons for our observed distribution remain unknown.

We thank Mr Marvin J. Podgor, of the Office of Biometry and Epidemiology of the National Eyc Institute, and Dr Andrew Schachat for their suggestions and guidance in preparing this paper.

This work was supported in part by the Retina Research and Development Fund, Philadelphia, the Pennsylvania Lions Sight Conservation and Eyc Research Foundation, Inc., and the Research Department. Wills Eye Hospital.

\section{References}

1 Gass JDM. Pathogenesis of disciform detachment of the neuroepithelium: part II. Idiopathic central serous chorioretinopathy. Am J Ophthalmol 1967; 63: 587-615.

2 Lyons DE. Conservative management of central serous retinopathy. Trans Ophthalmol Soc UK 1977: 97: 214-6.

3 Dellaporta A. Central serous retinopathy. Trans Am Ophthalmol Soc 1976; 74: 144-53.

4 Nanjiani M. Long-term follow-up of central serous retinopathy. Trans Ophthalmol Soc UK 1977; 97: 656-61.

5 Edwards JH. The recognition and estimation of cyclic trends. Ann Hum Genet 1961; 25: 83-7.

6 Terho EO. Lamm S. Heinonen OP. Scasonal variation in the incidence of farmer's lung. Int J Epidemiol 1980; 9: 219-20.

7 Sic-Boen-Lian. The etiological agent of central serous chorioretinitis. Ophthalmologica 1964; 148: 263-70.

8 McLean DM. Clinical and epidemiological aspects. Virology and health care, 1st ed. Baltimore: Williams and Wilkins, 1980): 15.

9 NIAID Task Force Report. Viruses causing acute respiratory diseases. In: US Department of Health. Education and Welfare. ed. Virology-acute viral infections. Bethesda. Maryland: PHS. DHEW No. (NIH) 79-1832, 1979; 2: 31-60. 